\title{
VERIFICAÇÃO DA INTEGRAÇÃO ENTRE O CADASTRO AMBIENTAL RURAL (CAR) E O CADASTRO NACIONAL DE IMÓVEIS RURAIS (CNIR) SOB A ÓTICA DO CADASTRO TERRITORIAL MULTIFINALITÁRIO
}

Luciana de Oliveira ${ }^{1}$

Francisco Henrique de Oliveira ${ }^{2}$

Resumo: O trabalho verificou a integração entre o Cadastro Ambiental Rural (CAR) e o Cadastro Nacional de Imóveis Rurais (CNIR) a partir da Lei de 'Georreferenciamento de Imóveis Rurais' (Lei 10.267/2001) e a Portaria Ministerial $n^{\circ} 511 / 2009$, que instituiu as diretrizes para o Cadastro Territorial Multifinalitário no Brasil. O CAR foi criado no novo Código Florestal (Lei 12.651/2012) e constitui-se numa base de dados georreferenciada sobre áreas florestais das propriedades privadas, com enfoque à preservação dos recursos florestais brasileiros no ambiente rural. Ainda em fase de implantação, o CAR tem demonstrado fragilidades em sua metodologia para levantamento geométrico dos limites a serem declarados, divulgando dados alarmantes a respeito de sobreposição de áreas. Por meio de uma proposta preliminar de um modelo conceitual, baseado no princípio teórico do OMT-G, verificou-se as discrepâncias e semelhanças conceituais, bem como o potencial de integração entre o Cadastro Ambiental Rural (CAR) e o Cadastro Nacional de Imóveis Rurais (CNIR), a fim de propor melhores práticas para a gestão territorial e ambiental. Como resultado obteve-se a caracterização conceitual e lógica sobre a menor unidade gerencial territorial de cada sistema cadastral (CAR e CNIR), bem como a respectiva comprovação das suas incompatibilidades. Assim, cada sistema gerencial espacial apresentou uma modelagem estruturante distinta que impede a potencial integração entre as bases de dados cadastrais $e$ amplia as divergências no reconhecimento e gerenciamento da unidade básica do território, quer seja para o planejamento ou para a tomada de decisão.

Palavras chave: Gestão territorial. Cadastro Ambiental Rural. Georreferenciamento de imóveis rurais.

\section{CHECKING OF INTEGRATION BETWEEN RURAL ENVIRONMENTAL REGISTER (CAR) AND NATIONAL REGISTER OF RURAL PROPERTIES (CNIR) CONSIDERING THE PRINCIPLES OF MULTIPURPOSE CADASTER.}

\begin{abstract}
The research verified the integration between the Rural Environmental Register (CAR) and the National Register of Rural Property (CNIR), based on the Law on 'Georeferencing of Rural Property' (Law 10.267 / 2001) and Ministerial Order No. 511/2009, which established the guidelines for the Multi-purpose Land Cadastre in Brazil. The CAR was created in the new Forest Code (Law 12.651 / 2012) and constitutes a georeferenced database on forest areas of private properties, focusing on the preservation of Brazilian forest resources in the rural environment. So far, it is in the implementation phase, the CAR

\footnotetext{
1 Mestre em Planejamento Territorial, Universidade do Estado de Santa Catarina, UDESC Centro de Ciências da Educação, Departamento de Geografia. Av. Madre Benvenuta, 2007. Itacorubi 88035001 - Florianópolis, SC - Brasil. luolive02@gmail.com, https://orcid.org/0000-0001-5640-7668

2 Professor, Universidade do Estado de Santa Catarina, UDESC Centro de Ciências da Educação, Departamento de Geografia. Av. Madre Benvenuta, 2007. Itacorubi 88035001 - Florianópolis, SC - Brasil. chico.udesc@gmail.com https://orcid.org/0000-0001-5884-5215
} 
has demonstrated weaknesses in its methodology for geometric survey of the limits to be declared, divulging alarming data regarding overlapping of the areas. Through a preliminary proposal of a conceptual model, based on the theoretical principle of the OMT-G, was verified the conceptual discrepancies and similarities, as well as the integration potential between the Rural Environmental Register (CAR) and the National Register of Rural Property (CNIR), in order to propose best practices for territorial and environmental management. As a result, the conceptual and logical characterization of the smaller territorial management unit of each cadastral system (CAR and CNIR) was obtained, as well as the respective proof of their incompatibilities. Each spatial management system presented a distinct structuring model that prevents potential integration between cadastre databases and widens the differences in the recognition and management of the basic unit of the territory, whether for planning or for decision-making.

Keywords: Territorial management. Rural Environmental Cadastre. Rural property georeferencing.

\section{VERIFICACIÓN DE LA INTEGRACIÓN ENTRE EL CADASTRO AMBIENTAL RURAL (CAR) Y EL REGISTRO NACIONAL DE INMUEBLES RURALES (CNIR) BAJO LA ÓPTICA DEL REGISTRO TERRITORIAL MULTIFINALITARIO.}

Resumen: El trabajo verificó la integración entre el Catastro Ambiental Rural (CAR) y el Catastro Nacional de Bienes Raíces (CNIR) a partir de la Ley de Georreferenciación de Bienes Raíces (Ley 10.267 / 2001) y la Orden Ministerial n 511/2009, que instituyó las directrices para el Catastro Territorial Multifinalitario en Brasil. El CAR fue creado en el nuevo Código Forestal (Ley 12.651 / 2012) y se constituye en una base de datos georreferenciada sobre áreas forestales de las propiedades privadas, con enfoque a la preservación de los recursos forestales brasileños en el ambiente rural. En el momento de la implantación, el CAR ha demostrado fragilidades en su metodología para el levantamiento geométrico de los límites a ser declarados, divulgando datos alarmantes sobre la superposición de áreas. Por medio de una propuesta preliminar de un modelo conceptual, basado en el principio teórico del OMT-G, se verificaron las discrepancias y semejanzas conceptuales, así como el potencial de integración entre el Catastro Ambiental Rural (CAR) y el Catastro Nacional de Bienes Raíces (CNIR), con el fin de proponer mejores prácticas para la gestión territorial y ambiental. Como resultado se obtuvo la caracterización conceptual y lógica sobre la menor unidad gerencial territorial de cada sistema catastral (CAR y CNIR), así como la respectiva comprobación de sus incompatibilidades. Así, cada sistema gerencial espacial presentó un modelado estructurante distinto que impide la posible integración entre las bases de datos catastrales y amplía las divergencias en el reconocimiento y gestión de la unidad básica del territorio, ya sea para la planificación o para la toma de decisión.

Palabras clave: Gestión territorial, Registro Ambiental Rural, Georreferenciación de inmuebles rurales 


\section{Introdução}

A participação do Brasil no alcance das metas brasileiras de biodiversidade, restauração da vegetação nativa e de redução da emissão de gases de efeito estufa, em vários compromissos internacionais assumidos pelo país, como o Desafio de Bonn (2018), o Acordo de Paris (2016), os Objetivos de Desenvolvimento Sustentável (2015) e a Convenção de Diversidade Biológica (1993), reforçam a necessidade da gestão de políticas públicas que viabilizem o cumprimento de tais compromissos (Coalizão Brasil Clima, Florestas e Agricultura, 2018).

Neste contexto, um dos instrumentos criado pelo novo Código Florestal Brasileiro a partir da Lei 12.651/2012 e que pode colaborar com esse enfoque é o Cadastro Ambiental Rural (CAR), criado no âmbito do Sistema Nacional de Informações sobre Meio Ambiente (SINIMA), regulamentado pela Instrução Normativa (IN) № 02/2014 do Ministério do Meio Ambiente, e que consiste num registro público eletrônico, de âmbito nacional, obrigatório para todos os imóveis rurais públicos e privados, assentamentos e de povos e comunidades tradicionais, com a finalidade de integrar as informações ambientais das propriedades e posses rurais compondo base de dados para controle, monitoramento, planejamento ambiental e econômico e combate ao desmatamento nos mais de 5,7 milhões de propriedades e posses rurais no país.

A área passível de cadastro, publicada pelo CAR, e estimada, com base no último censo realizado no país pelo IBGE, o Censo Agropecuário de 2006, contabiliza um total de 397.836.864 ha. Dados do último relatório do CAR, datado de 29 de maio de 2018 demonstram que já foi cadastrada uma área total correspondente a 448.319.254,00 hectares (ha), ou seja, $112,7 \%$ da área passível de cadastro divulgada. A mesma fonte divulga, porém em documento anterior (datado de 31 de maio de 2017), que a sobreposição com Terras Indígenas (TI's) é de 11.863.194,18 ha e corresponde a 6.379 imóveis; a sobreposição com Unidades de Conservação (UC's) é de 5.296.387,30 ha, correspondente a 37.169 imóveis; demonstrando claramente que há sobreposição de áreas cadastradas (SBF, 2017 e 2018).

Por tratar-se de um assunto recente e ainda, em fase de implantação, justificam-se estudos que proporcionem uma melhor e maior compreensão sobre a metodologia definida para se aplicar o Cadastro Ambiental Rural (CAR), promovendo uma visão de suas correlações/conseqüências com a atual estruturação cadastral rural brasileira no intuito de 
fomentar melhores práticas na utilização de ferramentas e instrumentos que contribuam com uma gestão territorial e, conseqüentemente, ambiental, eficientes.

\section{CADASTRO AMBIENTAL RURAL, O CADASTRO NACIONAL DE IMÓVEIS RURAIS E O CADASTRO TERRITORIAL MULTIFINALITÁRIO}

De acordo com a IN n²/2014, que define os procedimentos gerais para execução do Cadastro Ambiental Rural não há a exigência de um técnico e/ou profissional especializado para gerar o dado que comporá o banco de dados do sistema. Nesse sentido, é permitido que o próprio produtor rural ou qualquer pessoa leiga, maior de 18 anos e por ele autorizada, faça a planta cadastral do seu imóvel, definindo a delimitação do perímetro e das respectivas áreas de conservação diretamente sobre a imagem de satélite disponível no sistema (Rapid Eye 5m). Segundo Scolforo et al (2014), especialistas informam que esse método de delimitação das áreas disponibilizado no sistema é, entre todas as opções disponíveis, o menos preciso, uma vez que a escala das imagens é 1:50.000, essencialmente diferindo da potencial escala de visualização no módulo de cadastro que pode ser até a escala 1:5.000. Nesta situação, cada 1 (um) centímetro na imagem corresponde a 50 (cinquenta) metros na sua escala de delimitação. Desta forma acredita-se que a estratégia, criada com o intuito de facilitar a inscrição dos produtores, por outro, dificulta a validação do cadastro por falta de critérios técnicos e a criação de informações geométricas sobrepostas.

Dentre as iniciativas de criação de bases territoriais, o Brasil criou anteriormente ao CAR por meio da Lei no 5.868/ 1972, alterada pela Lei 10.267/2001 e regulamentada pelo Decreto 4.449/2002, o Cadastro Nacional de Imóveis Rurais (CNIR), gerenciado pelo Instituto Nacional de Colonização e Reforma Agrária (INCRA). Esta lei estabelece uma integração de informações com o cartório de Registro de Imóveis (RI) - Lei n 6.015/1973 e a exigência da identificação dos imóveis rurais através da identificação das coordenadas dos pontos que definem os pontos de inflexão do objeto e de mudança de confrontação, amarradas ao Sistema Geodésico Brasileiro e com uma precisão posicional pré-definida, melhor 50 centímetros para limites artificiais. (Brandão e Santos Filho, 2008).

O INCRA disponibiliza seus dados publicamente por meio do Sistema de Gestão Fundiária (SIGEF), sistema que foi criado para subsidiar a governança fundiária do território nacional. Através do SIGEF são efetuadas a recepção, validação, organização, regularização e disponibilização das informações georreferenciadas dos limites dos imóveis rurais, públicos e privados. Com o objetivo de garantir a segurança e a integridade 
das informações inseridas no sistema, os dados só podem ser enviados por profissional credenciado de formação técnica especializada. Após o envio dos dados, caso não seja detectada sobreposição ou qualquer outra irregularidade técnica, de acordo com o $§ 5^{\circ}$ do art. 176 da Lei 6.015/73, incluído pela Lei 11.952/09 o credenciado pode solicitar a certificação das áreas cadastradas.

Ainda recentemente o INCRA e a Receita Federal (RFB) assinaram em 31 de julho de 2017 a Instrução Normativa RFB n 1.725, que orienta os contribuintes sobre os novos procedimentos do Cadastro de Imóveis Rurais (CAFIR) da Receita Federal em virtude da nova coleta simplificada em conjunto com o INCRA para o adequado cumprimento das obrigações cadastrais perante o Imposto Territorial Rural, disponibilizando o CNIR para a coleta dos dados. O país avança na integração entre as informações territoriais, no intuito de garantir a segurança geométrica, jurídica e fiscal das propriedades rurais do país, possibilitando uma nova fase para a gestão territorial do país.

Outra iniciativa, e que corrobora com a visão multifinalitária do cadastro no país, foi a publicação da Portaria Ministerial n 511/ 2009, proposta pelo Grupo de Trabalho em Cadastro do Ministério das Cidades, que estabeleceu as diretrizes para a criação, instituição e atualização do Cadastro Territorial Multifinalitário (CTM) nos municípios brasileiros. Cadastro Territorial Multifinalitário que é definido aqui, segundo Brandão e Santos Filho (2008), como um inventário territorial sistemático e oficial do município e baseia-se no levantamento dos limites de cada parcela, e recebe um código identificador único.

As considerações sobre o CTM foram inspiradas nas recomendações da Federação Internacional de Geômetras - FIG: [...] e, portanto, um cadastro é normalmente baseado em parcelas e se caracteriza em um sistema de informação que contém registros de interesses sobre a terra, como direitos, restrições e responsabilidade. Usualmente inclui uma descrição geométrica das parcelas juntamente com outros registros que descrevem a natureza dos registros, a propriedade ou o controle desses interesses, e geralmente inclui o valor e suas benfeitorias. Pode ser estabelecido para propósitos fiscais (avaliação e impostos), propósitos legais (transferência), propósito social e para ajudar a gestão do território (planejamento e outros propósitos administrativos), permitindo um desenvolvimento sustentável e a proteção do meio ambiente (Cunha e Erba, 2010).

A partir do Século XX, a informação territorial do cadastro deixou de ser utilizada somente para fins fiscais e legais e passou a incluir vários outros interesses voltados ao objeto "parcela". Dessa forma, o cadastro territorial passou a ser contextualizado no novo 
cenário da multifinalidade e assume o papel de base para um sistema geral de informações territoriais. De forma gradativa, os conceitos de planejamento e gestão foram introduzidos sob o enfoque multifinalitário do cadastro, ampliando o seu uso para os setores público e privado, para planejamento territorial (urbano e rural), gestão territorial, gestão ambiental e desenvolvimento sustentável, entre outros (Cunha e Erba ,2010).

Assim sendo, remetendo-nos a questão da escala adotada pelo CAR, Cunha e Erba (2010) descrevem que o mapeamento sistemático nacional na escala 1:50.000 é utilizado atualmente no Brasil como a principal fonte de informações cartográficas, por ser homogênea e abrangente no território. Entretanto, deve-se considerar que, para essa escala, o erro geométrico atrelado restringe suas aplicações ao planejamento e aos estudos da organização espacial na esfera regional. Portanto, é um documento cartográfico que, em função do seu teor informacional, ou seja, escala pequena de representação das feições apresenta um erro geométrico posicional que não permite aplicação adequada aos objetivos do cadastro territorial multifinalitário.

Lane e Richards (1998), afirmam que é impossível administrar um país, estado ou município sem o conhecimento exato e fiel dos recursos naturais e econômicos disponíveis, os quais sejam representados em escala de detalhamento e correlação geométrica fidedigna com a realidade, o que é condição mínima indispensável para se chegar a gestão ambiental.

Desta forma, constatou-se a necessidade de uma avaliação técnica detalhada referente à modelagem conceitual e prática aplicada do Cadastro Ambiental Rural (CAR), bem como sua correlação com o Cadastro de Imóveis Rurais (CNIR).

A metodologia correlacionou técnica e geometricamente, as referências técnicas citadas do CAR e do CNIR, verificando o potencial de integração entre as bases de dados, com base nos preceitos levantados do CTM, utilizando comparação de suas unidades básicas de cadastro e modelagem conceitual para verificar se há integração ou não.

A comparação das unidades básicas de cadastro do CAR e do CNIR foi feita a partir do estudo da Legislação e Documentação Técnica associada, para a compreensão de sua definição e composição, bem como a comparação de suas geometrias disponíveis em suas bases de dados.

Já a correlação cadastral será feita a partir de uma proposição de modelagem conceitual preliminar, utilizando a linguagem OMT-G (Object Modeling Technique for Geographic Applications). 


\section{O MODELO DE DADOS OMT-G (OBJECT MODELING TECHNIQUE FOR GEOGRAPHIC APPLICATIONS)}

Segundo Silberchatz et. al (1999) um modelo de dados configura-se por um conjunto de ferramentas conceituais usadas para a descrição de dados, relacionamentos entre os dados, semântica de dados e regras de consistência.

De acordo com Borges, Davis Jr e Laender (2005) modelo de dados para aplicações geográficas possuem necessidades adicionais, tanto com relação à abstração de conceitos e entidades, quanto ao tipo de entidades representáveis e seu inter-relacionamento.

O modelo escolhido para ser usado nesse trabalho foi o OMT-G (Object Modeling Technique for Geographic Applications). Proposto inicialmente por Borges et al (2001), foi escolhido para este trabalho por constituir-se certamente em um dos principais modelos aplicados no país com um importante conjunto de trabalhos acadêmicos e técnicos como por exemplo, o documento Especificações Técnicas para Estruturação de Dados Geoespaciais Digitais Vetoriais da Comissão Nacional de Cartografia (EDGV/CONCAR), bem como adotado pela Infraestrutura Nacional de Dados Espaciais -INDE (RAMOS, 2016).

OMT-G é um modelo de dados orientado à objeto, dotado de recursos para o projeto de bancos de dados e aplicações geográficas. Parte das primitivas definidas para 0 diagrama de classes da Unified Modeling Language (UML), introduzindo primitivas geográficas com o objetivo de aumentar a capacidade de representação semântica daquele modelo e, portanto, reduzindo a distância entre o modelo mental do espaço a ser modelado e o modelo de representação usual.

Borges, Davis Jr e Laender (2005) descrevem que o modelo é baseado em três conceitos principais: classes, relacionamentos e restrições de integridade espaciais. As classes e relacionamentos definem as primitivas básicas usadas para criar esquemas estáticos de aplicação.

O modelo OMT-G divide as entidades modeladas em duas classes: georreferenciadas e convencionais. Por estas duas classes é possível representar, de maneira integrada, os três grandes grupos de fenômenos que ocorrem em geoprocessamento: os de variação contínua no espaço, os de variação discreta e os não espaciais. Os fenômenos de variação contínua constituem o que se convencionou chamar de geo-campos, e os de variação discreta constituem geo-objetos (BORGES, DAVIS E LAENDER, 2005). 
OMT-G propõe o uso de três diferentes diagramas no processo de desenvolvimento de uma aplicação geográfica. O primeiro e mais usual é o diagrama de classes, no qual todas as classes são especificadas junto com suas representações e relacionamentos. A partir do diagrama de classes é possível derivar um conjunto de restrições de integridade espaciais, que deve ser observado na implementação. Quando o diagrama de classes especifica múltiplas representações ou a derivação de uma classe a partir de outra, é necessário desenvolver um diagrama de transformação. Nele todo o processo de transformação pode ser especificado, permitindo a identificação dos métodos necessários para a implementação. Finalmente, para especificar as alternativas de visualização que cada representação pode assumir, é necessário desenvolver um diagrama de apresentação. O termo apresentação é usado no sentido de determinar o aspecto visual ou gráfico (envolvendo parâmetros como cor, tipo de linha, espessura da linha e padrão de hachura), de geo-objetos e geo-campos, no papel ou na tela do computador. (BORGES, DAVIS E LAENDER, 2005).

Este trabalho usou o Diagrama de Classes para verificação e proposição de integração entre o Cadastro Ambiental Rural e o Cadastro Nacional de Imóveis Rurais.

\section{Material e Métodos}

Para entendimento da unidade básica territorial entre o Cadastro Ambiental Rural (CAR) e o Cadastro Nacional de Imóveis Rurais (CNIR) foi feita inicialmente uma análise pautada na legislação que define o conceito de imóvel rural proposto nos dois cadastros, bem como uma comparação dos dados georreferenciados disponíveis em suas bases territoriais para visualização de suas similaridades e diferenças.

Os limites foram escolhidos aleatoriamente com relação a dois estados distintos que já possuíam um histórico maior na consolidação de suas bases de cadastro ambiental rural: os estados de São Paulo (SP) e Mato Grosso (MT).

Aqui entende-se que a questão quantitativa não é o principal objetivo e sim a qualitativa, propiciando a visualização das semelhanças e diferenças entre as unidades básicas territoriais utilizadas em cada base de dados cadastral, por isso a escolha foi aleatória e restrita.

Para tanto, utilizou-se inicialmente o sistema de informações geográficas opensource QGis, onde foi realizada a avaliação comparativa entre duas propriedades escolhidas aleatoriamente, porém ambas certificadas, tanto na base de dados do INCRA, 
órgão responsável pelas informações geométricas no CNIR, quanto na base de dados geométricos dispostos no CAR para verificação da correlação geométrica.

Os dados vetoriais do CAR estão disponíveis no SICAR, plataforma que integra as informações, que são gerenciadas estadualmente, para todo o Brasil, através do website <http://www.car.gov.br>, mediante consulta por 'Unidade Federativa' e posteriormente 'Município'. Os dados estão em formato shapefile com Coordenadas Geográficas e Datum SIRGAS 2000 (EPSG 4674). É possível desta forma fazer o download das áreas declaradas no CAR como: Área do Imóvel, APP (Área de Altitude superior a 1800m, Área Declividade maior que 45, Área Topo de Morro, Nascente Olho D’Água, Banhado, Borda de Chapada, Manguezal, Restinga), Reserva Legal, Área Consolidada, Área de Pousio, Hidrografia, Servidão Administrativa e Uso Restrito. Para esta análise foi utilizado apenas o dado temático correspondente a área do imóvel.

Os dados vetoriais do CNIR estão disponíveis no SIGEF, no website <http://www.sigef.incra.gov.br>, mediante consulta por 'Parcelas' que podem ser localizadas pelo CPF ou CNPJ do detentor, Nome do detentor, Código do Cartório, Número da Matrícula ou Transcrição, Código da Parcela, Protocolo de Envio, Código do Credenciado, Código do Vértice ou Código do Imóvel (SCNR/INCRA). Os dados estão em formato shapefile com Coordenadas Geográficas e Datum SIRGAS 2000 ou em formato KML com Coordenadas Geográficas e Datum WGS84 (EPSG 4326).

\section{Resultados e Discussão}

As Figuras 1 e 2 mostram o imóvel 'A' e 'B' e seus respectivos cadastros no SICAR e no SIGEF, onde percebe-se além das diferenças geométricas sobre os objetos, também se verifica a diferença conceitual sobre a menor unidade territorial utilizada como referência pelas duas bases de dados (sistemas).

No sistema do CAR a unidade de referência geométrica utilizada é de imóvel rural baseada no Estatuto da Terra e descrita conforme artigo 4’: 'I - "Imóvel Rural", o prédio rústico, de área contínua qualquer que seja a sua localização que se destina à exploração extrativa agrícola, pecuária ou agro-industrial, quer através de planos públicos de valorização, quer através de iniciativa privada. ' Assim conforme o artigo $2^{\circ}$, inciso $1^{\circ} \mathrm{e}$ artigos 31 e 32 da IN n²/2014 do MMA o imóvel rural é conceituado como 'uma ou mais propriedades ou posses contínuas pertencentes à mesma pessoa física ou jurídica, de 
direito público ou privado em regime individual ou comum que se destine ao uso comum e a preservação dos recursos naturais renováveis'.

Em outras palavras o CAR permite que mais de uma propriedade ou posse, em área contínua seja considerada como uma única inscrição para o imóvel e tem como referência de continuidade ou descontinuidade o conceito descrito no Código Civil onde quando um rio corta o imóvel rural, passagem forçada ou servidão legal de passagem e também estrutura viária que corta o imóvel rural (ferrovia, rodovia, estrada), não implica descontinuidade.

(a)

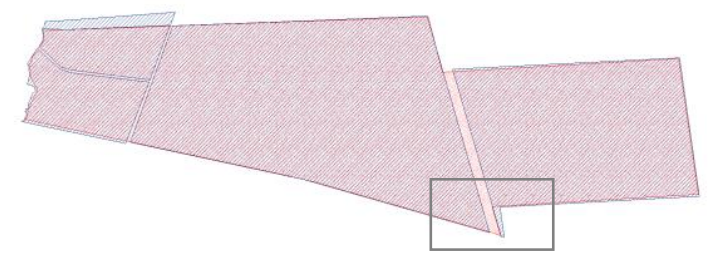

(c)

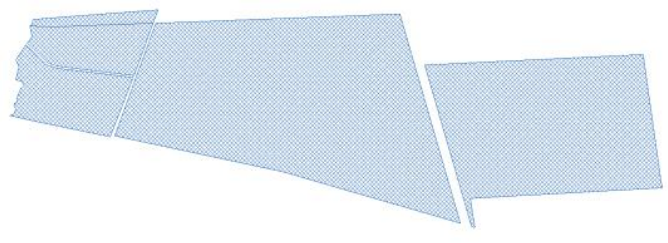

(b)

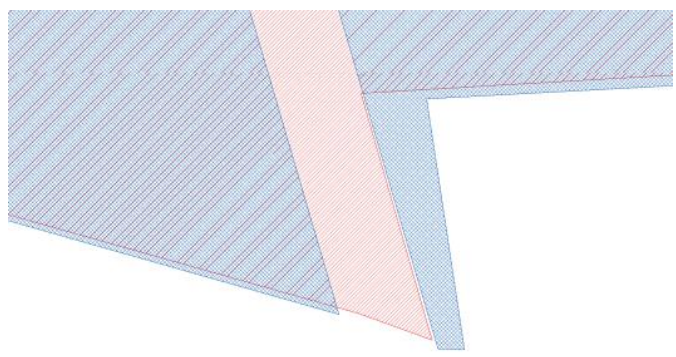

(d)

Figura 1: Exemplo de propriedade 'A' localizada no município de Bilac - SP.

(a)

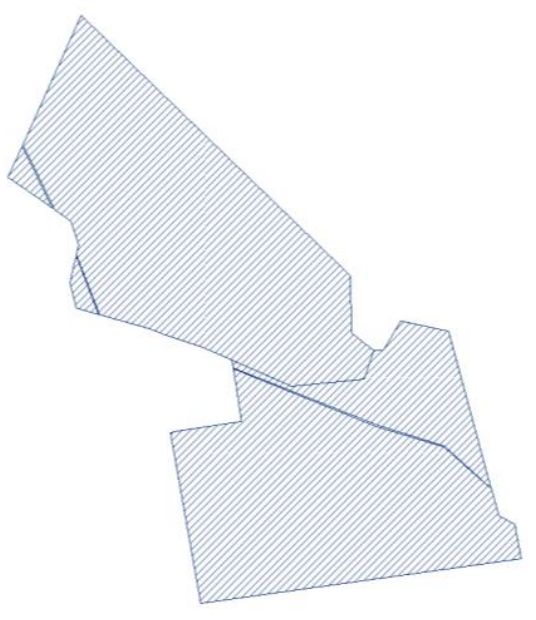

(b) 


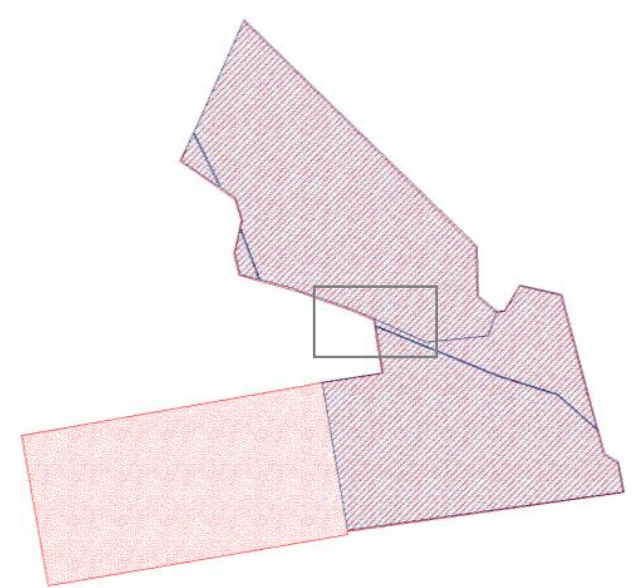

c)

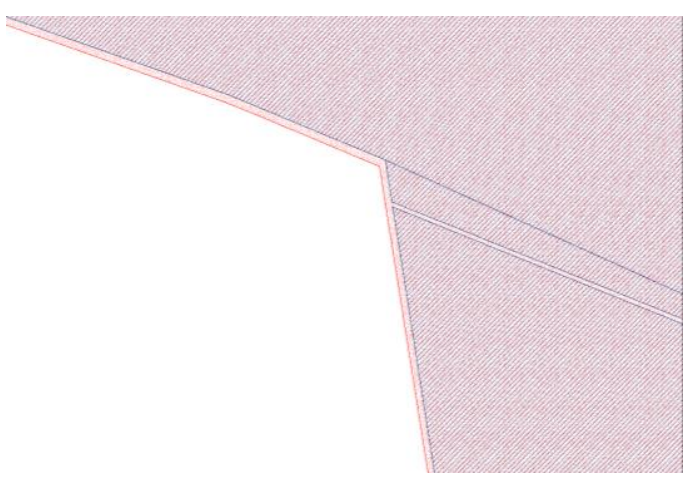

(d)

Figura 2: Exemplo de propriedade 'B' localizada no município de Lucas do Rio Verde - MT Em ambas figuras: (a) imóvel cadastrado no CAR (b) parcelas correspondentes ao mesmo imóvel cadastradas no CNIR/INCRA (c) sobreposição geométrica dos imóveis (d) detalhe da área destacada em (c) mostrando a incoerência geométrica entre os limites definidores dos objetos cadastrado pelos distintos sistemas.

Isso difere da unidade de referência geométrica, adotada pelo CNIR e também utilizada pelo CTM, onde a parcela cadastral é a menor unidade do cadastro definido como uma parte contígua e contínua da superfície terrestre com regime jurídico único. Assim não deve haver no banco de dados unidade menor que a parcela que devem ser contíguas, ou seja, não se sobrepor ou existir lacunas, e deve ser contínua, ou seja, sem interrupção por outra área dominial, com regime jurídico único (um único título legal ou condição de posse). O imóvel rural neste caso pode ser considerado composto por um modelado que se compõe por um conjunto de parcelas.

Desta forma observou-se que a unidade básica de cadastro do CAR e do CNIR tem conceitos diferentes não podendo serem modeladas, da forma que se encontram hoje, o que dificulta uma proposição de integração tendo como base o conceito da multifinalidade cadastral. Porém o trabalho demonstrou que, realizando algumas adaptações e considerações, foi possível a proposição de um modelo conceitual preliminar, conforme será demonstrado no capítulo seguinte.

\section{MODELO CONCEITUAL PRELIMINAR DE INTEGRAÇÃO COM OS DADOS DO CAR E CNIR}


Tomando por referência a lógica comparativa demonstrada anteriormente, fundamenta-se o princípio executivo de correlação entre os cadastros (CAR e CNIR), a partir da adoção dos dados brutos provenientes dos sistemas cadastrais supracitados.

Desta forma para haver uma equidade optou-se pela modelagem desenvolvida a partir da análise dos atributos referentes aos dados disponibilizados pelas bases de dados online do CAR, através do SICAR e do CNIR, através do SIGEF.

Da mesma forma que foi feito na análise da unidade básica de cadastro CAR e CNIR, o intuito da execução dessa etapa não foi quantificar, mas sim qualificar os dados disponíveis nos dois sistemas, SICAR e SIGEF, compreendendo a estrutura de classes e objetos com relação aos produtos (em formato vetorial) para a verificação de possível integração. Para tal, foi realizado o download dos dados disponíveis referente a um município aleatório primeiramente no SICAR <http://www.car.gov.br>, apresentados nas tabelas 1 e 2.

Tabela 1: Dados disponibilizados pelo SICAR

\begin{tabular}{l|l}
\hline Referência & Nome do Arquivo \\
\hline APP & APP.zip \\
\hline Área Altitude Superior & AREA_ALTITUDE_SUPERIOR_1800.zip \\
1800 & \\
\hline Área Consolidada & AREA_CONSOLIDADA.zip \\
\hline $\begin{array}{c}\text { Área Declividade Maior } \\
45\end{array}$ & AREA_DECLIVIDADE_MAIOR_45.zip \\
\hline Área Imóvel & AREA_IMOVEL.zip \\
\hline Área Pousio & AREA_POUSIO.zip \\
\hline Área de Topo de Morro & AREA_TOPO_MORRO.zip \\
\hline Banhado & BANHADO.zip \\
\hline Borda Chapada & BORDA_CHAPADA.zip \\
\hline Hidrografia & HIDROGRAFIA.zip \\
\hline Manguezal & MANGUEZAL.zip \\
\hline Nascente de Olho & NASCENTE_OLHO_DAGUA.zip \\
D'água & \\
\hline Reserva legal & RESERVA_LEGAL.zip \\
\hline Restinga & RESTINGA.zip \\
\hline Servidão Administrativa & SERVIDAO_ADMINISTRATIVA.zip \\
\hline Uso Restrito & USO_RESTRITO.zip \\
\hline Vegetação Nativa & VEGETACAO_NATIVA.zip \\
\hline Vereda & VEREDA.zip \\
\hline
\end{tabular}

Após o download foi construída a tabela que apresenta a Relação de Classes e Objetos (RCO) correspondentes a classe ÁREA_IMÓVEL. Em programação orientada a objetos, conforme Couto (2017) a RCO tem por finalidade explicar as nomenclaturas das 
classes e suas descrições (dicionário de dados), atributos, tipos de dados que definirão os tipos de informações que podem ser inseridas em um determinado campo (int, double, char, varchar, date e boolean), tipo de geometria adotado para representar a classe de objeto e elementos herdados de outras classes, constituindo-se em uma etapa necessária anterior à modelagem.

Tabela 2: Análise dos atributos referentes aos dados de Área do Imóvel

\begin{tabular}{|c|c|c|c|c|c|c|}
\hline Coluna & $\begin{array}{l}\text { Tipo de } \\
\text { Dado }\end{array}$ & $\begin{array}{l}\mathbf{N}^{\circ} \\
\text { Registro } \\
\text { s Nulos }\end{array}$ & $\begin{array}{l}\text { № Máximo de } \\
\text { algarismos/car } \\
\text { acteres }\end{array}$ & $\begin{array}{l}\text { Valor } \\
\text { Mínimo }\end{array}$ & $\begin{array}{l}\text { Valor } \\
\text { Máximo }\end{array}$ & $\begin{array}{l}\text { Frequência } \\
\text { Máxima dos } \\
\text { Registros } \\
\text { únicos }\end{array}$ \\
\hline COD_IMOVEL & varchar & 0 & 100 & $\begin{array}{l}\text { AC- } \\
\text { 1200013- } \\
0018 F 3109 \\
93 \mathrm{D} 476 \mathrm{~B} 90 \\
57721347 \mathrm{E} \\
21 \mathrm{E} 38\end{array}$ & $\begin{array}{l}\text { AC- } \\
\text { 1200013- } \\
\text { FFEE77E } \\
\text { F329B47 } \\
\text { 90BB05B } \\
\text { C040DDA } \\
\text { F89A }\end{array}$ & 1 \\
\hline NUM_AREA & double & 0 & 31 & 1,057295 & $\begin{array}{l}147012,3 \\
77864\end{array}$ & 2 \\
\hline COD_ESTADO & varchar & 0 & 100 & AC100 & $\mathrm{AC}$ & 1 \\
\hline NOM_MUNIC & varchar & 0 & 100 & Acrelândia & $\begin{array}{l}\text { Acrelândi } \\
\text { a }\end{array}$ & 1 \\
\hline NUM MODULO & double & 0 & 31 & 0,0106 & 86,0781 & 6 \\
\hline TIPO IMOVEL & varchar & 0 & 3 & IRU & AST & 2608 \\
\hline SITUĀCAO & varchar & 0 & 2 & $\mathrm{CA}$ & AT & 2332 \\
\hline CONDICAO_I & varchar & 0 & 85 & $\begin{array}{l}\text { Analisado } \\
\text { com } \\
\text { pendências } \\
\text { ' } \\
\text { aguardand } \\
\text { atendiment } \\
\text { o a outras } \\
\text { restrições }\end{array}$ & $\begin{array}{l}\text { Aguardan } \\
\text { do análise }\end{array}$ & 760 \\
\hline
\end{tabular}

Com relação aos dados disponíveis no SIGEF, é possível fazer o download do arquivo referente ao limite da parcela nos formatos shapefile, KML ou CSV.

Tabela 3: Dados disponibilizados pelo SIGEF:

\begin{tabular}{l|l}
\hline Referência & Nome do Arquivo \\
\hline parcela & codigo_parcela.zip \\
\hline
\end{tabular}

Tabela 4: Análise dos atributos referentes aos dados de parcela (SIGEF)

\begin{tabular}{l|l|l|l|l|l|l}
\hline Coluna & $\begin{array}{l}\text { Tipo de } \\
\text { Dado }\end{array}$ & $\begin{array}{l}\mathbf{N}^{\circ} \\
\text { Registro } \\
\text { s Nulos }\end{array}$ & $\begin{array}{l}\text { № Máximo de } \\
\text { algarismos/car } \\
\text { acteres }\end{array}$ & $\begin{array}{l}\text { Valor } \\
\text { Mínimo }\end{array}$ & $\begin{array}{l}\text { Valor } \\
\text { Máximo }\end{array}$ & $\begin{array}{l}\text { Frequênci } \\
\text { a Máxima } \\
\text { dos } \\
\text { Registros } \\
\text { únicos }\end{array}$ \\
\hline qrcode & varchar & 0 & 255 & $\begin{array}{l}\text { bc58c75c- } \\
84 a 7-44 c 2- \\
\text { a283- }\end{array}$ & $\begin{array}{l}\text { bc58c75c- } \\
84 a 7-44 c 2- \\
\text { a283- }\end{array}$ & 1 \\
\hline
\end{tabular}




\begin{tabular}{l|l|l|l|l|l|l}
\hline & & & & $\begin{array}{l}\text { 3d6254933 } \\
\text { 3d3 }\end{array}$ & $\begin{array}{l}\text { 3d6254933 } \\
\text { 3d3 }\end{array}$ & \\
\hline nome & varchar & 0 & 255 & $\begin{array}{l}\text { Matrícula } \\
1432\end{array}$ & $\begin{array}{l}\text { Matrícula } \\
1432\end{array}$ & 1 \\
\hline
\end{tabular}

A proposta preliminar do modelo conceitual apresentado na Figura 3 foi construída de acordo com as primitivas apresentadas pelo modelo OMT-G, já descritas nesse trabalho, utilizando a ferramenta OMT-G Designer disponível on-line por meio do link http://aqui.io/.

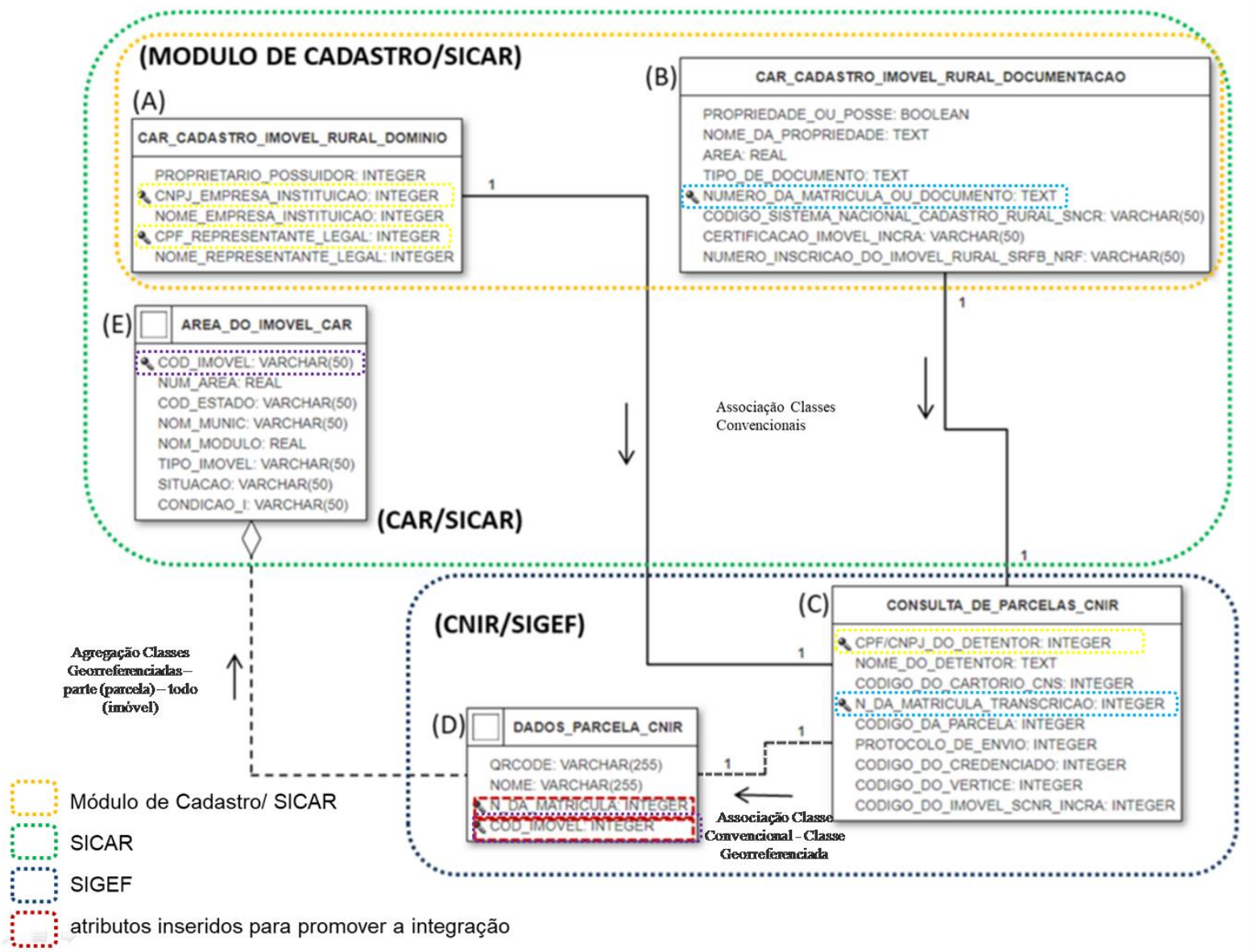

Figura 3: Modelagem Conceitual preliminar proposta para integração entre o CAR e o CNIR a partir de unidade básica territorial.

Fonte: a Autora

A Figura 3 mostra dentro do quadro verde as tabelas disponíveis no SICAR, sendo as tabelas (A) e (B), dentro do quadro amarelo, são tabelas provenientes do "MÓDULO DE CADASTRO", item "CADASTRO DE IMÓVEL RURAL".

No quadro azul estão as tabelas provenientes do SIGEF a partir da interface de consulta de parcelas. 
Na tabela (A), disponível no subitem "DOMINIO" verificou-se a possibilidade de integração utilizando o dado requerido do "CPF REPRESENTANTE LEGAL" ou "CNPJ EMPRESA/INSTITUIÇÃO".

Da mesma forma, com a combinação da tabela (B), disponível no subitem "DOCUMENTAÇÃO" verificou-se a possibilidade de integração utilizando o dado requerido correspondente ao "NÚMERO DA MATRÍCULA".

Assim realizou-se uma integração entre as classes convencionais "CAR_CADASTRO_DE_IMOVEL_RURAL_DOMINIO"

“CAR_CADASTRO_DE_IMOVEL_RURAL_DOCUMENTACAO” com a classe convencional “CONSULTA_DE_PARCELAS_CNIR”, utilizando um Relacionamento de Associação com Cardinalidade de 1 para 1, indicando que para cada entrada da combinação das chaves "CPF REPRESENTANTE LEGAL" ou "CNPJ EMPRESAINSTITUIÇÃO" com a chave "NUMERO_DA_MATRICULA" é possível vincular-se a uma parcela, menor unidade territorial do cadastro do CNIR, desde que previamente cadastrada no SIGEF.

Uma observação para tornar mais eficaz essa vinculação entre os dois cadastros é que tanto para os dados "CPF/CNPJ" como para o dado "NUMERO_DA_MATRICULA", é recomendado que estejam no mesmo formato. No cadastro do SIGEF, tanto o dado "CPF/CNPJ" quanto o dado "NUMERO_DA_MATRICULA" são definidos no padrão INTEGER.

A partir da parcela identificada relaciona-se através do Relacionamento de Associação uma classe convencional a uma classe georreferenciada "DADOS_PARCELA_CNIR", tabela (D), sendo a geometria em formato shapefile da unidade básica territorial desta base de dados.

Por sua vez a classe georreferenciada "DADOS_PARCELA_CNIR" conecta-se à classe georreferenciada "AREA_DO_IMÓVEL_CAR" através do relacionamento Agregação, sugerindo que uma ou mais parcelas podem compor o imóvel.

\section{Considerações Finais}

A questão de utilizar a parcela como a unidade básica territorial baseia-se na análise realizada a respeito da metodologia, disponível no SIGEF a partir dos documentos como a Norma Técnica para Georreferenciamento de Imóveis Rurais (3ª Edição), conjuntamente com o Manual Técnico de Limites e Confrontações (1a Edição) que fornecem subsídios satisfatórios para o melhor registro geométrico da parcela cadastral em vigência hoje no 
Brasil, corroborando com a visão multifinalitária, em considerar superfície contínua e contígua, não permitindo desta forma sobreposições ou espaçamentos entre seus registros.

Sabe-se que como o CAR permite que qualquer pessoa realize o seu georreferenciamento, a entrada de dados não disponibiliza a visualização de dados dos vizinhos para confrontação. Apesar de hoje ainda não cobrir todo o território e não realizar cadastro de posses, o cadastro das parcelas territoriais do CNIR seria uma entrada de dados já certificada, diminuindo esforços e custos ao cadastrante do CAR, incentivando sua regularização fundiária, conectando os cadastros rurais.

Mesmo não se configurando uma base de dados responsável pela garantia da propriedade, o CAR, como base de dados ambientais, está atrelada a dados territoriais e espaciais, portanto sendo executado a partir de uma metodologia que não garante a confiança geométrica na definição dos limites, reproduzindo uma 'realidade' equivocada enquanto as informações ambientais geradas, produzindo dados inconsistentes com figuras geométricas distintas sobre o mesmo objeto.

Os exemplos verificados neste trabalho mostram que podem ocorrer desde pequenas diferenças como grandes diferenças entre os limites cadastrados na base do SICAR, quando comparado com o mesmo objeto cadastrado na base do SIGEF, fato que se extrapolado para nível nacional geram preocupantes diferenças de áreas, bem $\mathrm{cm}$ sobreposições, mostrando um exemplo de desintegração cadastral que interfere tanto na credibilidade do sistema, quanto na geração de uma morosidade de validação dos dados.

A modelagem preliminar proposta nessa pesquisa considera como base estruturante a fundamentação teórica do cadastro territorial apregoado pela FIG e corrobora com o modelo adotado pelo CNIR, tornando explicita que a estrutura do cadastro do CAR encontra-se aquém do que se espera para ser estabelecido um sistema gerencial territorial baseado na estrutura e reconhecimento da unidade parcelária.

O modelo sugerido não tem a pretensão de se encerrar nele mesmo, porém traz à tona a necessidade de discussão e de maior integração entre os entes federais/estaduais/municipais gestores do reconhecimento e (re) ordenamento territorial. A partir da apresentação da lógica desenvolvida nesse modelo preliminar, tem-se claro que novos modelos e/ou versões serão estabelecidos e amadurecidos, tomando por foco a mesma linha de raciocínio administrativo territorial parcelário.

Dentro disso, uma outra opção a ser verificada é a utilização do código SNCR. Este código numérico, composto por 13 dígitos no formato 999.999.999.999-9, é atribuído pelo 
INCRA a cada imóvel rural e está presente também na tabela do CAR como dado de entrada da documentação do imóvel. Para a geração desse código o INCRA baseia-se na definição de imóvel rural que constitui-se por “(...) área formada por uma ou mais matrículas de terras contínuas, do mesmo titular (proprietário ou posseiro), localizada tanto na zona rural quanto urbana do município. O que caracteriza é a sua destinação agrícola, pecuária, extrativista vegetal, florestal ou agroindustrial”. Essa definição se aproxima mais da definição de imóvel rural utilizada pelo CAR, oriunda do Estatuto da Terra conforme já citado neste trabalho, definição essa que considera o conceito de continuidade, porém não o de contiguidade, e agrupa sobre o mesmo CPF propriedades adjacentes do mesmo titular. Porém, da mesma forma, gera dúvidas quanto a essa agregação, já que afirma corresponder a propriedades localizadas também na área urbana, sabendo que o CAR, abrange por sua vez, apenas propriedades localizadas em área rural.

Os exemplos de cadastro demonstrados nesse trabalho, bem como o de outras iniciativas cadastrais criadas no Brasil, apenas reforçam o cenário caótico da realidade fundiária brasileira, que promovem bases cadastrais desarticuladas, ocasionando não só justaposição e inconformidade geométrica, bem como redundância de informações, desperdício de tempo e investimentos, dificultando a existência de uma base de dados cadastral com subsídio a gestão territorial integradora e de caráter multifinalitário.

A informação ambiental, por ser um objeto territorial, poderia utilizar-se de base fundiária já existente, base essa certificada e validada a partir de critérios técnicos que garantem uma maior confiabilidade posicional, como a do INCRA, compondo base temática ambiental consistente como subsídio a planejamento, gestão e monitoramento.

O Brasil teria um enorme ganho no reconhecimento territorial da sua malha fundiária (rural e urbana) se fosse efetivamente definido um órgão oficial gestor e que terá a fonte de dados oficializada para se tornar referência aos demais atores da sociedade.

\section{REFERÊNCIAS}

BORGES, K.; DAVIS JR, C.; LAENDER, Alberto. Capitulo 3 - Modelagem Conceitual de dados geográficos IN Bancos de Dados Geográficos. Ed. Mundo Geo. Curitiba - PR, 2005. Disponível em: http://www.dpi.inpe.br/livros/bdados/cap3.pdf acessado em 21 de janeiro de 2018.

BORGES, K. A. V., DAVIS JR., C. A., LAENDER, A. H. F. OMT-G: An Object-Oriented Data Model for Geographic Applications. Geoinformatica, v. 5, n. 3, p. 221-260, 2001. 
BRANDÃO, A.C. E SANTOS FILHO, A. V. Sistema de Cadastro Territorial Georreferenciado em Áreas Urbanas. Revista Ver a Cidade - Ano 3 - no 3 - maio de 2008. Disponível em http://www.veracidade.salvador.ba.gov.br/v3/images/veracidade/pdf/artigo5.pdf acessado em 02 de outubro de 2016.

BRASIL. Instrução Normativa $n^{\circ} 2$ - MMA, de 06 de maio de 2014. Dispõe sobre os procedimentos para a integração, execução e compatibilização do Sistema de Cadastro Ambiental Rural-SICAR e define os procedimentos gerais do Cadastro Ambiental Rural CAR. Disponível em http://www.car.gov.br/leis/IN_CAR.pdf acessado em 07 de maio de 2017.

BRASIL. Lei no 12.651 , de 25 de maio de 2012. Dispõe sobre a proteção da vegetação nativa; altera as Leis nos 6.938, de 31 de agosto de 1981, 9.393, de 19 de dezembro de 1996, e 11.428, de 22 de dezembro de 2006; revoga as Leis nos 4.771, de 15 de setembro de 1965, e 7.754, de 14 de abril de 1989, e a Medida Provisória no 2.166-67, de 24 de agosto de 2001; e dá outras providências. 2012. Disponível em: http://www.planalto.gov.br/ccivil_03/_ato20112014/2012/lei/l12651.htm acessado em 15 de abril de 2016.

COALIZÃO BRASIL CLIMA, FLORESTAS E AGRICULTURA. Validação do CAR: realidade e desafios. Disponível em https://www.huffpostbrasil.com/coalizao-brasil-clima-florestas-eagricultura/validacao-do-car-realidade-e-desafios_a_23218293/ acessado em 24 de junho de 2018.

COUTO, Rodrigo de Souza. Estruturação, Modelagem e Implementação de Banco de Dados Geoespaciais para o Cadastro Ambiental Rural. Dissertação de Mestrado apresentada ao Programa de Pós-Graduação em Geociências Aplicadas do Instituto de Geociências da Universidade de Brasília. Brasilia -DF, 2017. Disponível em http://repositorio.unb.br/bitstream/10482/24640/1/2017_RodrigodeSouzaCouto.pdf acessado em 03 de fevereiro de 2018.

CUNHA, E.M.P. E ERBA, D.A. (Org.). Manual de Apoio - CTM: Diretrizes para a criação, instituição e atualização do cadastro territorial multifinalitário nos municípios brasileiros. Ministério das

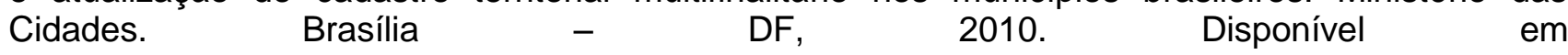
http://www.capacidades.gov.br/media/doc/acervo/c4924c559c0b1b95a8ad38c47fda4799.pdf acessado em 02 de outubro de 2016.

INCRA - Instituto Nacional de Colonização e Reforma Agrária. Norma Técnica para Georreferenciamento de Imóveis Rurais. 3a Edição. Ministério do Desenvolvimento Agrário Instituto Nacional de Colonização e Reforma Agrária. Nov, 2003.

INCRA - Instituto Nacional de Colonização e Reforma Agrária. Manual Técnico de Limites e Confrontações. 1a Edição. Ministério do Desenvolvimento Agrário - Instituto Nacional de Colonização e Reforma Agrária. Nov, 2003.

INCRA - Instituto Nacional de Colonização e Reforma Agrária. Estatísticas de Imóveis Rurais. Cadastro de Imóveis Rurais - Brasil. (atualizado em junho 2016). Disponível em http://www.incra.gov.br/sites/default/files/uploads/estrutura-fundiaria/estat-sticas-de-im-veis-rurais/brasil_cadastro_imoveis_rurais_geral_pub_e_priv.pdf acessado em 16 de junho de 2017

LANE, S.N.; RICHARDS, K.S. Landform Monitoring Modelling and Analysis. New York, John Wiley \& Sons. 1998.

RAMOS, J. A. S.; Nota Técnica: Banco de dados geográficos para a gestão ambiental municipal. IN Gestão e governança local para a Amazônia sustentável: notas técnicas - 2 / Orgs. Maria Cristina Soares de Almeida e Peter H. May. - Rio de Janeiro: IBAM, 2016. Disponível em: http://www.amazonia-ibam.org.br/biblioteca/publicacao/detalhe/gestao-e-governanca-local-para-aamazonia-sustentavel---notas-tecnicas---volume-02/144/sim acessado em 21 de janeiro de 2018. 
SCOLFORO, J.R., CAMPOS, S., BORGES, L.A.C., MORAES FILHO, L.O., SANTOS, P.A., ABREU, E.C.R., COUTO JÚNIOR, A.C.S., NASCIMENTO, R.C., OLIVEIRA, A.L., BARROS, D.A., LAUDARES, S.S., PEREIRA, C.M. Noções de Geotecnologias. Curso de Extensão à Distância. Curso de Capacitação para o Cadastro Ambiental Rural (CapCAR). UFLA - Universidade Federal de Lavras. Lavras: 2014.

SFB - Serviço Florestal Brasileiro. CAR - Cadastro Ambiental Rural - Boletim Informativo - Dados até 31 de maio de 2017. Disponível em http://www.florestal.gov.br/documentos/car/boletim-docar/2799-boletim-sicar-ate-31-maio-2017/ file acessado em 16 de junho de 2017.

SFB - Serviço Florestal Brasileiro. CAR - Cadastro Ambiental Rural - Boletim Informativo - Dados até 29 de maio de 2018. Disponível em http://www.florestal.gov.br/documentos/car/boletim-docar/3657-boletim-informativo-edicao-especial-4-anos-car/file acessado em 24 de junho de 2018.

SILBERCHATZ, A.; KORTH, H. F.; SUDARSHAN, S. Sistema de Banco de Dados. São Paulo: Pearson Makron Books, 1999 3를 edição 904p.

\section{NOTAS DE AUTOR}

\section{CONTRIBUIÇÃO DE AUTORIA}

Luciana de Oliveira - concepção, coleta de dados, análise de dados, elaboração do manuscrito, discussão dos resultados.

Francisco Henrique de Oliveira - participação da discussão dos resultados; revisão e aprovação da versão final do trabalho.

\section{FINANCIAMENTO}

Não se aplica

\section{CONSENTIMENTO DE USO DE IMAGEM}

Não se aplica

\section{APROVAÇÃO DE COMITÊ DE ÉTICA EM PESQUISA}

Não se aplica

\section{CONFLITO DE INTERESSES}

Não se aplica

\section{LICENÇA DE USO}

Este artigo está licenciado sob a Licença Creative Commons CC-BY. Com essa licença você pode compartilhar, adaptar, criar para qualquer fim, desde que atribua a autoria da obra.

\section{HISTÓRICO}

Recebido em: 31-05-2018

Aprovado em: 13-07-2018 